\title{
Handbook of Primary Care Ethics
}

Edited by

Andrew Papanikitas and John Spicer

First published 2018

ISBN: 978-1-4987-6967-9 (hbk)

ISBN: 978-1-3151-5548-7 (ebk)

\section{Chapter 4}

Compassion in primary and community healthcare

Joshua Hordern

(CC BY 4.0)

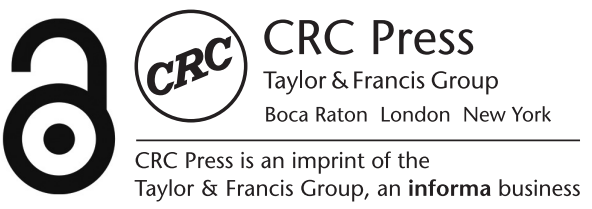




\section{Compassion in primary and community healthcare}

JOSHUA HORDERN

Compassion in primary and community healthcare

Compassion for patients in plural polities

Power, critique and this 'secular' time
Time, compassion and justice

30

25

Conclusion: Fostering compassion

31

Acknowledgements

32

References

\section{COMPASSION IN PRIMARY AND COMMUNITY HEALTHCARE}

Compassion is an attribute of a person's affective understanding, which aims to enable, so far as possible, shared experiences of the world's ills and some alleviation of those ills' effects. Such an attribute is thus of great value within healthcare institutions such as general practices and other primary and community healthcare settings. It may characterise the people who participate in those institutions; or, it may not so characterise them. The appearance of compassion, under certain conditions and even in fragile and incomplete forms, is a kind of human excellence, a way of being for the good in community. Compassion is not, therefore, a commodity, to be bought, sold and traded. Although time can be costed, there is no line for compassion in any budget. Were compassion to be thought a commodity, one could imagine trading it off against some more measurable factor (efficiency, cost-effectiveness, etc.). However, our human capacity for compassion, though fragile, tends to resist such marginalisation and reductionism.

As an attribute of human affective understanding aiming at shared experience amidst life's illness, compassion is cognitive, participative and alleviative. As an affection, compassion is not reducible to mere sensation, although it may coincide with physical expressions such as weeping or reassuring touch. Rather, compassion is centrally an affective attitude towards someone's suffering, a core dimension of the 'partnership-working' crucial to patient-practitioner relationships in the 'new professionalism' of health and social care. ${ }^{1}$ The affectivity of compassion does not entail that it is somehow non-rational or anti-rational. Rather, compassion is directed towards situations, people or things with which it is concerned. This directedness or 'aboutness' of compassion indicates that we can ask questions about its intelligibility and reasonableness - whether understanding of an individual's suffering is being appropriately grasped and communicated in this case. This ability to assess compassion strongly suggests

\footnotetext{
* For this account of fragile virtue, see Ref. 2.
} 
that it is in some way cognitive, involving a kind of belief or mental attitude. Precisely as cognitive, it can initiate reasoning towards action which will constitute the alleviation of suffering.

If this is so, compassion cannot be reserved or detached. Compassion alleviates suffering by participating in it. As an essentially alleviative affection, it reaches out in understanding and embodied service to engage with persons in need. In compassion, one person relates their self to the other, seeking to share in understanding of that other's experience of the suffering human condition.* Just because of its quality as a kind of intelligent understanding rather than an inscrutable sensation, compassion may constitute an experience which is intelligibly shared by both sufferer and carer, leading to shared decision and action. However, this participative, alleviative sharing in suffering is not automatic. It is a goal to be achieved in the everyday encounters of healthcare.

Since compassion is like this, its content is not fixed but filtered through the circumstances and beliefs of those concerned. Accordingly, compassion is quite different from a healthcare professional's self-indulgent emoting, which signals his or her own self-regard instead of aiming at shared experience. More emotion does not equate to better compassion; ill-focussed emoting is worse than detached but efficient practice. However, intelligent, well-directed affection can enable practice that is humane and effective.

This description of compassion focusses compassion not chiefly on specific practical manifestations of concern important though they are - the accessibility of an elderly person's home, the practicability of contacting a general practitioner (GP) out of hours and the glass of water within reach - but on persons' deeper experiences of illness, disease and care. Participation by compassion in that deeper level is cognitive and affective, and only thus can it be alleviative. For the logic of compassion is that suffering involves loss of some goodness in human life. In healthcare, alleviation of suffering might not involve action beyond kindly presence aimed at reassurance of solidarity and relief of fear: the health visitor or GP having that second cup of tea with the lonely person so that their story is properly heard. Or, it may involve extensive activity aimed at diagnosis and healing of disease. However, both of these forms of alleviation are initiated by an affective, cognitive participation in suffering, aiming at the shared experience both of suffering and of some goodness by the one who has lost or never known it.

Compassion is, therefore, not an optional extra but a necessary and in some fashion everpresent quality of healthcare, central to its goals. To construe the term 'concordance', a term which complements 'compliance' or 'adherence', in terms of the moral psychology of shared decision-making, compassion involves being united affectively with another in their experience

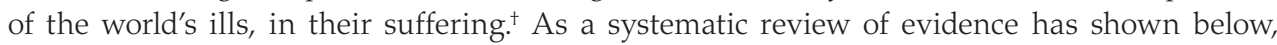
such 'emotional rapport and support [are] ... associated with improvements in emotional health,

\section{Elements of compassion}

- Cognitive-affective - We understand each person's experience of suffering, listening in love.

- Participative - We seek, so far as possible, to share that experience, being bodily present in service.

- Alleviative - We think about how to make things better, looking forward in hope.

- Persuasive - We talk, helping one another understand suffering differently and better.

- Civic - We encounter each other as citizens in 'secular' solidarity, being alert to power, justice and culture.

\footnotetext{
* For this account of affectivity in more detail, see Refs. 3 and 4.

+ For a brief introduction to these terms, see Ref. 5 .
} 
symptom resolution, physical functioning and quality of life assessments, as well as ... measures ... such as physiological indicators of disease management ... and pain control' ${ }^{6}$

Primary and community healthcare's practices such as listening, visiting, discussing, advising, comforting, recommending, prescribing and referring concern encounters between persons amidst life's drudgery, drama and hypochondria. Persons engaging in such everyday practices encounter one another in ways which bear witness to their profound human unity amidst vulnerability and suffering. For these practices enflesh human solidarity as somebody inclines ear, mind and whole person towards somebody else in time of need. Through these practices, primary and community healthcare (hereafter 'primary care') workers often act as 'gatekeepers' to healthcare services: as district nurses identifying medical needs among the housebound, occupational therapists enabling someone to access help needed for daily tasks and GPs discerning whether specialist care is appropriate.

Primary care, characterised by its generalism and by these specific practices of encounter, is the beginning of a road down which patients may travel, a hospitable entry point on a journey towards specialist avenues of care they may require. ${ }^{7}$ The task of primary care is then a demanding one, requiring clinical understanding leavened with compassion so that the vast undifferentiated mass of human distress and suffering ${ }^{\prime 8}$ may be ordered, personalised and then alleviated. To continue welcoming sufferers' faces in the all-too-brief encounters of countenances that embody compassion requires perseverance, not only through each long day but also, where continuity of care is maintained, over the long years during which trust may grow amidst the sometimes frightening but often fertile trials of life.

\section{COMPASSION FOR PATIENTS IN PLURAL POLITIES}

In practice, the character of encounters between primary care workers and patients cannot be generalised beyond a certain level because compassion is for this person at this time and because forms of primary care vary. Nonetheless thinking about compassion can be disciplined by understanding its nature as irreducibly concerned with personal subjects seeking shared experience and understanding. In many present-day societies, a complication arises. Although in traditional societies, a relative homogeneity of culture was common, in modern nation-states, this has been widely, though not universally, replaced with overlapping, intersecting networks of diverse, heterogeneous cultures. These multiple cultures nonetheless share a common political identity and, in many European contexts at least, a socialised healthcare system, albeit to varying degrees. Health, being 'a basic socio-personal good', 9 valued variously by all cultures, therefore provides a point of reference in political life, in which the beliefs and practices of plural societies can meet in disciplined conversation.

Since health is both social and personal, compassion is necessarily a civic matter as an individual's suffering becomes a matter of public concern, mediated through the persons of primary care workers. However, since present-day civic life is plural, a new understanding of compassion in primary care is needed. Those who seek to exercise compassion, as a form of affective, cognitive, participative understanding aimed at alleviating subjective suffering and attentive to cultural factors, must grasp the diverse ways that individual and community values concerning health and illness shape people's outlook. Sharing experience involves the ability to identify imaginatively ${ }^{\prime 10}$ with the way that a particular patient perceives their condition and its significance.

Patient perception will of course include expectations about what should be done, if anything, about their condition, a trend entrenched by access to medical information 
online and 'shared decision-making'." More generally, personalisation of healthcare relating to

a patient with respect, at least ideally, means treating their experiences, perceptions and preferences not just as relevant data for professional decision-making but as matters worth taking seriously in their own right including within decision-making partnerships. ${ }^{11}$

However, taking patient perceptions seriously does not mean that compassion involves uncritical affirmation of such perceptions. One might be tempted to think this if one failed to grasp compassion's affective, cognitive, participative, alleviative nature and role in decision-making. Such a failure would make it harder to say 'no' to a patient for it allows compassion to become mere acquiescence not only to a patient's perception but also to that patient's demands. However, for two reasons, this uncritical notion of compassion as acquiescence should be resisted.

Firstly, while a person's condition is their condition and they are in an important sense an expert in it - it is their experience and no one else's ${ }^{\dagger}$ - conditions are not absolutely unique because persons are not absolutely unique. Persons are members of the species of persons, characterised by observable regularities of health and disease. Those who have knowledge of such regularities, formulated as evidence which then informs the cognitive understanding that constitutes compassion, are better placed than those who do not to assess diagnoses, interpretations and expectations regarding health conditions and so to resist, kindly and respectfully, certain patient perceptions and demands.

Secondly, a patient's perception of their condition and best interests may be challenged, gently and transparently, in order for the alleviative dimension of compassion to be realised. Compassion's cognitive quality is ordered to seeking all patients' good, from those patients who are demanding and confident about their wishes to those who do not grasp or care about their interests to those who, for example, feel that nicotine or alcohol use is all that makes life bearable but are afraid to clash with the official view, represented by the doctor, that such behaviour is in some way wrong. In short, how a patient perceives their circumstances may have much to do with culturally mediated or individually constructed values and beliefs which may require open, if tender, challenge.

While the paternalistic 'doctor/nurse knows best', presupposition is rightly no longer prevalent partly due to a diminution of unstudied deference in society, it is also right that primary care provides a context in which patient perceptions of their situation can be kindly, respectfully, gently and transparently but critically discussed. Inasmuch as compassion involves a cognitive participation in the feelings of another - in particular their experience of suffering - then, compassion must incorporate this element of critical analysis and even persuasion, whereby suffering may be interpreted by both patient and healthcare practitioner differently and better. Primary healthcare workers' 'power to do good' and 'relational expertise' amount to social authority to know and pursue goodness even amidst the flat landscape of liberal political life that tends to discourage others' 'interference' in the lives individuals are building or destroying for themselves. ${ }^{12}$ Such a landscape may, without the wise mediation of primary care workers, end up populated by people abandoned to loneliness and ill health. ${ }^{\ddagger}$

\footnotetext{
* For a multi-perspectival analysis of this trend, see Ref. 13. For discussion of how technology will and perhaps should displace the face-to-face encounter in parts of healthcare, see Ref. 14

+ For weaknesses in the idea of the 'expert patient', see Ref. 15.

$\ddagger$ As one commentator on Edmund Pelligrino's work puts it, 'One cannot abandon persons to their autonomy when they are in difficult straits'.16
} 


\section{POWER, CRITIQUE AND THIS ‘SECULAR' TIME}

Yet, there is a dark side to compassion's critical dimension in which persuasion or encouragement becomes domination and oppression and in which professional power undermines the patient's own reasoning and ignores their interests. Beware the idealisation of the doctor as moral saint! Humanities disciplines, such as theology, have been at pains to analyse the fragility of individuals' moral quality and unmask the self-deceptiveness which attends status and power.

Moreover, cultural wisdom garnered through engagement with patients and their cultures may itself function to critique a professional's own prejudice or vice and improve the way he or she understands suffering and compassion. However, far from diminishing the social authority of healthcare professionals, this two-way street in compassion actually reinforces their role as mediators in between and advocates for the individuals and cultures they serve and indeed between those cultures and professional, regional or government policy. This is a position of influence in which primary care workers are called to moral discernment in critical service of the population among whom they practice. Cribb and Gewirtz note this critical edge and its relation to justice when they comment that healthcare practitioners:

... will need an awareness that respecting the autonomy of individuals, by trying to respond to their needs and preferences, is not only potentially in conflict with beneficence to that individual, but may also exact some 'cost' on wider groups or populations - and thus may be in tension with other important concerns such as social justice or population effectiveness. ${ }^{17}$

The possibility of critique signals a continuing professional development need for those in primary care. For while the individual encounter is basic to general practice, wider cultural factors require primary care workers' critically compassionate understanding. Culture is of course not simply local, especially in the digital age where beliefs and communities are formed online in dispersed networks. Nonetheless, local cultural practice remains a decisive influence on the lives of many. GP practices and community healthcare teams, such as church ministers, local authority councillors and national politicians, typically have some sense of defined geographical responsibility. Just so, primary healthcare workers must mediate between local and cultural expectations regarding health and what is practically possible, bearing in mind regional and national factors. On this point at least, there is a similarity between the account here and 'values-based practice' as 'a less prescriptive and more local approach [which] aims to introduce a greater variability of viewpoints and greater recognition for individually specific values. ${ }^{\prime 18}$

To deploy a term in its traditional rather than its contemporary usage, this culturally astute compassion may be best dubbed 'secular', not at all in the sense of being 'anti-religious' but rather in its native theological sense in which 'secular' is a word for a Christian idea - that of the quality of the time in this age, when ultimate questions of the meaning of human life have not been finally answered and when cultures live side by side in shared 'penultimate' civic life. The 'secular', on this view, is the time in which diverse philosophies, theologies, religions, values and moral outlooks contribute respectfully and critically to a plural society's public good. Inasmuch as time allows space for conversation, the secular may be thought of as the forum for differing forms of thought to meet in sometimes critical conversation. ${ }^{*}$ Compassion, understood

\footnotetext{
* See e.g. Ref. 19.
} 
as a cognitive, affective, critical, alleviative participation in suffering, is the proper beginning of the moral understanding of suffering, shaping discourse, deliberation and policy, a basic feature of this secular time in plural polities.

Thus, what is required is a morally substantial, affectively rich notion of 'secular' citizenship and discourse, embedded within professional training and development and benefitting society at large. Primary and community healthcare services, rooted in locality and conversant with that locality's cultures, are well placed to cultivate such civic discourse as the context in which compassion may be richly practised and experienced. A primary healthcare worker's understanding of and participation in local cultural life are key to the realisation of compassion in practice and so a proper focus for ongoing professional education. Inasmuch as deep understanding of multiple cultures is not itself a cultural norm, primary care workers must be countercultural if they are to be compassionate. They must be places of resistance to ways of perceiving cultural difference which are characterised by either impatient ignorance or uncritical acquiescence.

\section{TIME, COMPASSION AND JUSTICE}

Compassion has now been considered in terms of time in the sense of the secularity of the age in which political identity is now constituted. However, compassion also concerns time in the equally down to earth but more intuitive sense that time available to participate compassionately in suffering is scarce as primary care workers move from patient to patient and home to home. Time is often too pressured to engage in depth with each person encountered, share properly in decisions or pursue 'the search for meaning'. ${ }^{20}$

An important factor shaping this experience is the way that time and compassion are bound up with justice. One attitude to their interrelation is 'to perceive healthcare rationing problems as involving an explicit opposition between justice and caring ${ }^{\prime 21}$ GPs in particular increasingly have responsibilities for considering how the needs of each one should be justly related to the needs of the very many.* The daily challenge appears in the widely attested experience of 'no time' or 'very little time' for compassion. Ten-minute (or briefer) appointments seem inadequate to create shared experience between patients and practitioners, the goal towards which compassion aims: a man with heart disease and depression, the teenager only needing a prescription and the domiciliary visit which is just 'social'. Although continuity of care may mitigate this problem, the dearth of time is a constant challenge which requires transparency in dialogue so that issues which the patient wants to address come into the open. The primary care worker can aid this transparency by honestly specifying how few minutes are available, thus focussing the conversation supportively rather than foreclosing it and avoiding difficult dialogue by handing out a prescription or by some other means.

However, filling the day to capacity militates against the kind of care which is required and leads to an 'appointment book that lies to us about the properties of time', precisely in its

\footnotetext{
* Carlsen and Norheim suggest that this resource allocation responsibility involves significant challenges to maintaining a compassionate practice, as patients become 'demanding consumers' or 'shared decision-makers', thus reshaping - perhaps unhelpfully - societal respect for healthcare workers' professional standing (Ref. 22). Over against the changing nature of general practice, Iona Heath mounted a passionate argument in favour of general practitioners' 'partisan' advocacy for patients and against such doctors taking financial responsibility for allocation of scarce resources. For, so Heath argued, the doctor's very responsibility for allocation will be understood by patients as a threat and so undermine the trust necessary for his or her relationship with the doctor (Ref. 23).
} 
deceptive 'visual representation of time, setting out the day in appointment-sized chunks', ${ }^{24}$ which do not measure up to patient needs. That which is visible, the schedule of appointments, attempts to give structure to the underlying shared purpose upon which the patientpractitioner relationship is premised, that purpose of making space for compassionate shared experience. However, a lack of or misuse of time may result in failing to reach the goal of shared experience and so do injustice to some in favour of others. In this situation, time spent which is not answerable to a metric or budget, is the time which it may feel harder to justify.

This scarcity paradigm regarding compassion bears witness to important truths: that time is limited and that the GP's appointment book and its equivalents in other forms of primary care are full of a weight of human need that stretches the capacities and waking hours of healthcare workers to breaking point. And yet, the paradigm may occlude the way that compassion is a participative, cognitive, alleviative affection. Compassion is not a 90 -second feature of a 10-minute appointment in which one asks an 'extra' question about the patient's experience or family situation. Such a question is important in enabling shared understanding but compassion is not hermetically sealed within that segment of an appointment or visit. Rather, as an attribute of understanding, compassion may infuse the whole encounter, focussing attention in listening, assessment and diagnosis, consideration of the proper use of time and resources and engagement in underlying personal or cultural factors. Compassion also properly includes a justice consideration which can grasp that another patient further down the list will suffer if the current appointment goes on too long. Thus, compassion is not in opposition to justice - a thoughtless implication of the scarcity paradigm - but rather a constitutive feature of the realisation of justice. For compassion, as a cognitive, alleviative affection, is competent to understand not only suffering but also any injustice which may cause further suffering.

\section{CONCLUSION: FOSTERING COMPASSION}

For compassion to be realised in practice requires a supportive organisational ethos. Compassion is certainly basic to the shared experiences of patients and healthcare workers in primary care settings. However, such experiences should be supported by compassion between primary care colleagues. For those who care for the suffering are themselves vulnerable members of the human community. The GP who provides continuous long-term care to a local community may journey with the population through the challenges which life inevitably throws up, sharing something of his or her own life and suffering in a way which benefits patients. ${ }^{25}$ Similarly, the experience of sharing vulnerability prudently with those with whom one works can support compassionate practice. Endurance in compassion towards patients will be enabled by a shared concern for colleagues, which is intelligent, participative and alleviative, renewing and refreshing collegiality on a regular basis.

Such supportive workplace cultures rarely happen by accident and are easily endangered by the commodification of one's colleagues as units of production - efficient and lauded or inefficient and stigmatised. Teams in general practices, district nursing or health visiting, for example, need to sustain a compassionate ethos if people are to see colleagues in depth, building long-term, stable, working relationships. Each primary care worker needs regular personal refreshment, deep drinking from some well of meaning and purpose to be sustained for the next stage of the journey.

Offered as one such source of refreshment in a plural polity, the motto of the Royal College of General Practitioners, cum scientia caritas, emphasises the greatest of the 'three theological virtues', love. It stands as a reminder to those in primary care of what Health Education 
England's Simon Gregory called the need 'to regain, or not be afraid to admit to, our love of our patients'. ${ }^{26}$ Caritas, in its theological sense, speaks of the participative, merciful and hopeful love of God as revealed in Jesus Christ, enabling humanity's peaceful friendship with God and others. Such love serves sick and lonely persons that they might be accompanied in suffering and made better. Such love seeks this mercifully, never allowing the ascription of fault, however just, to stand in the way of that care and company. Informed by this caritas, the real knowledge of primary care may become true wisdom. This is love which deploys up-todate clinical evidence, seeks justice, rejoices in mercy and shows critical sensitivity to locality and culture. It is a love which, participating in the suffering human condition, becomes that intelligent compassion that unites in solidarity patients, professionals, managers and policymakers, fellow travellers all on the journey through the world's real sorrows, deep fears, enduring hopes and great joys.

\section{ACKNOWLEDGEMENTS}

This work was supported by the University of Oxford Wellcome Trust Institutional Strategic Support Fund (grant number 105605/Z/14/Z); and the Arts and Humanities Research Council (grant number: AH/N009770/1). The author gratefully acknowledges this funding and also that of the Sir Halley Stewart Trust. The views expressed within this chapter are those of the author and not necessarily those of the Sir Halley Stewart Trust.

\section{REFERENCES}

1. Cribb A and Gewirtz S. Professionalism. Cambridge: Polity Press; 2015. p. 33.

2. Adams RM. A Theory of Virtue: Excellence in Being for the Good. Oxford: OUP, 2006.

3. Hordern J. Political Affections: Civic Participation and Moral Theology. Oxford: OUP; 2013. Chapters 1-2.

4. Hordern J. What's wrong with 'compassion'? Towards a political, philosophical and theological context. Clin Ethics 2013; 8(4): 91-7.

5. Bell JS, et al. Concordance is not synonymous with compliance or adherence. Br J Clin Pharmacol 2007; 64(5): 710-11.

6. Roter DL, et al. Effectiveness of interventions to improve patient compliance: A metaanalysis. Med Care 1998; 36(8): 1138-61.

7. Papanikitas A and Toon P. Primary care ethics: A body of literature and a community of scholars? J R Soc Med 2011; 104: 94-6.

8. Heath I. The Mystery of General Practice. London: Nuffield Provincial Hospitals Trust; 1995, 27.

9. Maxwell B. Just compassion: Implications for the ethics of the scarcity paradigm in clinical healthcare provision. JME 2009; 35: 219-23.

10. Heath, 1995, op. cit., 35.

11. Cribb and Gewirtz, 2015, op. cit., 36.

12. Cribb and Gewirtz, 2015, op. cit., 56, 66-7, 97.

13. Edwards A and Elwyn G, editors. Shared Decision-Making in Health Care: Achieving Evidence-Based Patient Choice. Oxford: OUP; 2009.

14. Susskind R and Susskind D. The Future of the Professions: How Technology Will Transform the Work of Human Experts. Oxford: OUP; 2015. 
15. Badcott D. The expert patient: Valid recognition or false hope? Med Health Care Philos 2005; 8(2): 173-8.

16. Thomasma D. Establishing the moral basis of medicine: Edmund D. Pellegrino's philosophy of medicine. J Med Philos 1990; 15(3): 245-67.

17. Cribb and Gewirtz, 2015, op. cit., 81.

18. Petrova M, et al. Values-based practice in primary care: Easing the tensions between individual values, ethical principles and best evidence. Br J Gen Pract 2006; 56(530): 703-9.

19. Biggar N. Why religion deserves a place in secular medicine. JME 2015; 41(3): 229-33.

20. Heath, 1995, op. cit., 20.

21. Maxwell B. Just compassion: Implications for the ethics of the scarcity paradigm in clinical healthcare provision. JME 2009; 35: 219-23.

22. Carlsen B and Norheim O. 'Saying no is no easy matter'. A qualitative study of competing concerns in rationing decisions in general practice. BMC Health Serv Res 2005; 5: 70.

23. Heath, 1995, op. cit., 42-4.

24. Senior T. The strange time-bending properties of the appointment book. Br J Gen Pract 2014; 64(625): 416.

25. Malterud K, et al. When doctors experience their vulnerability as beneficial for the patients. Scand J Prim Health Care, 2009; 27: 85-90.

26. Gregory S. William Pickles Lecture 2014: Cum Scientia Caritas compassion with knowledge. Br J Gen Pract 2015; 65(630): 36-7. 
